\title{
Analysis of Urgent Neurologic In-Hospital Consultations in A Large Tertiary Hospital Center in China
}

\author{
Jiafang Wang ${ }^{1}$, Ming Ren ${ }^{2}$, and kebin Zeng ${ }^{3}$ \\ ${ }^{1}$ Department of Neurology, Affiliated Hospiatal of Chongqing Three Gorges Medical \\ Colloge, Chongqing \\ ${ }^{2}$ Affiliation not available \\ ${ }^{3}$ First Affiliated Hospital of Chongqing Medical University
}

April 29, 2020

\begin{abstract}
Objectives: To analyze the current condition of the urgent neurologic in-hospital consultations, which may help junior doctors to manage diseases related to neurology and carry out emergency consultation. Methods: We conducted a retrospective study of urgent neurologic in-hospital consultations in a large tertiary hospital for four consecutive years (Jan 1, 2014 to Dec 31, 2017). Through the electronic medical record system, the clinical data of patients who received in-patient consultation in emergency neurology department were reviewed and analyzed. Results: A total of 1,437 cases (age range 9 to 103 years old with average age 60.3 -year old, Male $54.6 \%$ ) were included in the study, $57.5 \%$ of cases met the urgent consultation criteria. The departments applying for urgent consultation involved 29 clinical departments. The most common reasons of urgent consultation were disturbance of consciousness (36.0\%), tic/stiffness (13.6\%), limb weakness (8\%) and mental disorders (5.6\%).Urgent consultation diseases were divided into three categories: neurological diseases $(77.8 \%)$, non-neurological diseases (10.4\%), and unknown diseases (11.8\%).Common neurological disorders were urgent cerebrovascular disease (33.6\%), epilepsy/status epilepticus (15.8\%), and metabolic or infection, toxic encephalopathy (14.9\%). Conclusions: Most cases of the urgent neurologic consultation met the urgent neurologic consultation criteria. Non-neurologic junior physicians should clinically enrich their knowledge of neurological diseases.
\end{abstract}

\section{Introduction}

Neurology is a branch of medicine concerned especially with the structure, function, and diseases of the nervous system including the central and peripheral nervous systems. To a great extent, the practice of Neurology depends on the knowledge of neuroscience and complex nervous system, which is very difficult for students to learn. Therefore, most students think that neurology is a complex subject. According to a survey, neurological difficulties are common not only among students, but also among older doctors[1,2]. It has been proposed that neurology and neurological diseases are special, so it challenges non neurologists, medical students and junior doctors[3,4], but also junior physicians.

Acute and severe diseases in neurology are characterized by rapid onset and progression. If the disease is not correctly diagnosed and treated in time, there will be more complications and higher mortality. Therefore, it is reported that the demand for neurology exceeds the supply $[5,6]$. The rapid and accurate diagnosis and adequate treatment are essential to improve the prognosis of acute nervous system diseases [7]. The main purpose of emergency consultation in neurology department is to help the consultation department deal with life-threatening symptoms in time, so as to improve the cure rate and quality of life of patients, the incidence rate and mortality rate are also reduced. However, the serious shortage of neurophysicians will affect the prognosis of the disease, which may be more serious in the future[6]. In fact, the disharmony 
of doctor-patient relationship also further aggravates the proportion of emergency consultation, which is a huge burden for neurologists. There were few reports about emergency consultation in neurology before. We conducted in-depth investigation on this.

\section{Methods}

\subsection{Subjects}

In this study, we collected the clinical cases of non neurological department inpatients who applied for emergency consultation in neurology department between Jan 1, 2014 and Dec 31, 2017 in the First Affiliated Hospital of XXX, which is a 3200-bed tertiary university hospital center in China.

\subsection{Methods}

For emergency consultation, the doctor applying for consultation should first contact the consultant of neurology department by telephone, and fill in the electronic case application form, including name, age, gender, number of inpatients, number of beds, specialty, reason and applicant. The invited consultant should arrive at the applicant ward within 10 minutes after received the phone. The urgent consultation was often conducted by the chief resident in neurology department from Monday to Friday, and by attending physicians on weekends and holidays. For some cases to be difficult to deal with, the young physicians should consult with the senior attending physician on duty.

After examining the data of the following patients, the neurologist should perform a neurological examination and then propose a diagnosis, further examination and treatment plan. For patients with multiple consultations, the final consultation conclusion was the final diagnosis in the study, which was obtained from the patient's hospital discharge records. This study analyzed the purpose of consultation, the spectrum of consultation diseases, the distribution of non-neurological disease departments, and the percentage of compliance with emergency consultation criteria.

There were no reports of emergency consultation criteria for neurology. Therefore, we had proposed the criteria for emergency consultation in neurology as follows: (1) stroke within 24 hours; (2) cases of critical illness requiring the assistance of the neurological physicians; (3) acute gas poisoning within 24 hours requiring emergency hyperbaric oxygen treatment; (4) other new emerging neurological disease symptoms, neurological signs and imaging abnormalities; (5) recurrent symptoms with poor therapeutic; (6) patients to be emergency surgery who had neurological diseases previously for preoperative evaluation; (7) cases with medical disputes.

\subsection{Statistic analysis}

In this study, excel office software was used for data collection and tabulation. The countable data values were expressed as mean \pm SD. Date processing was done using SPSS statistics version 23.0.

\section{Results}

\section{General information}

There were 1618 cases of urgent neurological in-hospital consultations in 4 years, 573 cases in 2014, 406 cases in 2015, 382 cases in 2016 and 257 cases in 2017 respectively. 136 cases had been consulted at least twice. But, only 1 case was counted for the same patient in the analysis of data. Therefore, 1437 cases were included in the final analysis, among which $54.6 \%$ were males and $45.4 \%$ were females. The average age was 60.3 years old (range from 9 to 103 years old).

\section{Consultation department involved}

The urgent neurologic consultation involved 29 clinical departments, 990 cases in the general ward and 447 cases in the intensive care unit (ICU). There were $68.3 \%$ cases from the department of internal medicine and the surgical departments.. The most common department applying for urgent consultation in the internal medicine department were department of emergency (12\%), departement of respiration (11.9\%), department 
of cardiology (11\%), department of hematology (7\%) and department of nephrology (6\%). The most common departments applying for urgent consultation in surgical department were department os thoracic surgery $(13 \%)$, orthopaedics (12\%), vascular surgery (11\%), hepatobiliary surgery (10\%) and ICU surgery $(10 \%)$.

\section{Purpose or Diseases of consultation}

The common purpose of inviting urgent neurologic in-hospital consultations are as following: diagnosis unknown (80\%), assisted treatment (14\%) and dispute cases (6\%) (Table 1). Other rare reasons for applying for urgent consultation are shown in the Table 1.

Table 1.

The diseases in the urgent neurologic in-hospital consultation can be divided into three categories: nervous system diseases, non-nervous system diseases and unknown diseases. The frequently neurologic consultation diseases are acute cerebrovascular disease, epilepsy/epileptic status, metabolic, infectious toxic ischemic and hypoxic encephalopathy, and intracranial infection, which account for $58.4 \%$ of the total urgent consultations. The older age, the more frequently common applied for urgent consultation. Non-neurological diseases accounted for $10.4 \%$ of the total urgent neurologic consultations, among which disturbance of internal enveriment, poor basic diseases and anxiety states were more common. Unknown diseases accounted for $11.8 \%$, which were due to many reasons including the complicated diseases to be known and patients' rejection to further test for the patient.

\section{Discuss}

Neurology is a difficult subject with many critical diseases for most of physicians. It is also a blind spot and difficult area in medical diagnosis and treatment. Therefore, urgent consultation in neurology department is very common in the tertiary hospital center. In this analysis, there are two main findings listed. First, neurological diseases account for $77.8 \%$ of total cases in the urgent consultation diseases, which is different from the disease spectrum of inpatients applying for emergency consultation in neurology department reported recently [1]. $57.5 \%$ of diseases in the urgent consultation were in line with the urgent consultation criteria. . Second, the most common reasons of urgent consultation were disturbance of consciousness, tic/stiffness, limb weakness and mental disorders. Common neurological disorders were acute cerebrovascular disease (33.6\%), epilepsy/status epilepticus, and encephalitis, metabolic or infective toxic encephalopathy.

In 1437 cases of urgent consultation, the actual number of cases was decreased year by year from 573 cases in 2014 to 257 cases in 2017, which may be attributed to the improvement of the clinical skills of nonneurologists in our hospital and the rational and effective management from the hospital medical services section. In this urgent consultation indications assessment, only $57.5 \%$ of consultations were in line with the urgent consultation criteria. Therefore, non-neurological practitioners should strengthen the learning of neural diseases; strictly make a judgment on the urgent neurological consultation. And medical manage office should put forward the appropriate management measures to enhance the effective urgent consultaionn.

In urgent consultation, acute cerebrovascular diseases account for $33.6 \%$, including cerebral infarction, cerebral hemorrhage and transient ischemic attack, which was similar with foreign reports [8]. In our hospital, the average age of patients with urgent consultation was 60.3 years old. This older age may be related to the highly incidence of stroke onset increasing with age [6]. Most of the consultations are in line with the guidelines of urgent consultation. However, the urgent consultation cases were decreased. There are several reasons for that. First, the management office has strengthen the importance of basic medical knowledge and basic skills. There are lots of training held during the continuing medical education. Second, the physicians are more skilled in making a diagnosis and treatment the acute cerebrovascular diseases. Third, there is a standardized clinical pathway for the physician to reference on several neurological diseases. There were 177 cases of epileptic/epileptic persistent state, which was different from the common urgent consultation diseases reported in domestic emergency consultation reports. The main reason may be that there is an epileptic center in our department, which is one of the famous epileptic centers in China. There are several experts on epilepsy and a large number of regular follow-up patients, which come from neighbor area with the poor 
treatment managed by non-specialist physicians before. The metabolic or infective toxic encephalopathy and encephalitis were the third largest group of urgent neurological consultation in our hospital. There are many patients with encephalopathy and encephalitis, who were treated for a longer time. In the hospital, there is a Chongqing Key Laboratory of Neurology, which is operated by full-time personnel. They can detect acid-fast bacillus with more than $80 \%$ positive results and abscissile cells. They also can detect more than ten antibodies related to autoimmune encephalitis, which are highly specific and sensitive. The neurological physicians could practice better management in making a diagnosis and treatment of encephalitis.

Some of departments had frequently applied for urgent neurological consultation including intensive care unit (ICU), respiratory medicine, cardiovascular medicine, hematology and nephrology. At present, it is estimated that there are about $10 \%$ of patients presented with some neurological manifestations [10-12]. It had been reported that the neurological consultation in ICU was often urgent consultation, which also suggested that the patients had server disease to be managed. At the same time, there are some of ICU doctors lacked relevant knowledge of neurologic critical illness. It advised that resident on internal medicine should be trained in neurological specialty at least 6 months with the 3 years of standardized resident training in China. In addition, physicians in the ICU should take part in more lectures on emergency neurology in our hospital. Most of the patients have a common occurrence of cerebrovascular disease in the department of cardiovascular medicine and nephrology, who have the risk factors of vascular sclerosis. Patients with renal failure are consulted by neurology because of the impaired consciousness or convulsive in dialysis, which are due to the internal environment dysfunction, renal encephalopathy and dialysis encephalopathy. The common reasons for the application for consultation in the department of respiratory medicine include convulsions, disturbance of consciousness, and the etiology is mostly pulmonary encephalopathy, ischemic and hypoxic encephalopathy. First of all, it is very important for them to control the primary disease. If the patients with lung tuberculosis presented with headache, disturbance of consciousness, convulsion, hyponatremia, the tuberculosis meningitis should be considered. And then the patients should be prescribed routine with the lumber puncture for cerebrospinal fluid analysis, dynamic electroencephalogram and brain enhanced MRI examination. Once the patients have been made a diagnosis of tuberculosis meningitis, physicians should prescribe with anti-tuberculosis drugs in combination at enough doses as soon as possible. The main reasons for applying for urgent consultation in surgical department are impaired consciousness. The uncontrollable postoperative epilepsy is one of the special reasons for the neurosurgery department. However, the preoperative evaluation and secondary prevention of cerebrovascular disease are often met in the surgical department, which should be consulted at regular time rather than an urgent consultation.

To take the accuracy case history and the neurologic examination may help the physician in the differential neurological diseases with others. There were 150 cases of non-neurological diseases including restlessness, mental disorder, disturbance of consciousness and headache, which were considered to be related to internal environmental disorders, primary diseases, hypertension, anxiety state and vagal reflex syncope. Some physicians confuse with the aphasia and unclear utterance in mental disturbance. Therefore, in order to reduce unnecessary urgent neurological consultation, physicians should rule out the above diseases before application for an urgent enological consultation. Acute encephalopathy often is secondary to infection after cardiopulmonary resuscitation or metabolic diseases. To control the primary causes is the main measures. Before applying for consultation, it is suggested for the physicians to complete blood gas analysis, electroencephalogram, blood glucose and electrolyte, computer tomography of the skull, and to take the detail history of drug use including sedatives and antidepressants. In the case of urgent consciousness disorder and cognitive impairment after surgery, drugs adverse effects should be considered first. Therefore the physicians should be familiar to the adverse effects of anesthetic drugs. For the tetanus/convulsion, as one of the common reason for urgent consultation, the physician should be familiar with the possible cause of the convulsion including the diseases of respiratory medicine, cardiology and other internal medicine. Only parts of them are diagnosed as epilepsy, which should apply for neurological consultation. Limb weakness is the same with them. Non-neurological diseases accounted for 10.4\%. Both internal medicine and surgical departments account for half of them, such as respiratory, cardiology, orthopedics, obstetrics and gynecology, and digestive departments. Non-neurologic diseases usually presented with irritability and delirium with no focal nervous 
system signs, which may be regarded as the differential criteria with the neurological diseases. Most of the consultation patients with non-neurologic diseases have no new structural damage in the central nervous system [13], and the treatment is mainly to maintain the stability of the internal environment for the treatment of primary diseases. Pain is a common cause of consultation in previous reports [14]. Some of them should be consulted with neurosurgical physician rather than neurologic physician such as cerebral contusion and laceration, skull base fracture, diffuse axonal injury, intracranial tumor, hydrocephalus, subdural hematoma, etc. The physicians should know more about neurosurgical knowledge.

Some of the consultations should be ordinary consultation rather than urgent consultation. For example, a patient with Parkinson's disease applied for emergency consultation in neurology department because asked for the intravenous treatment of ganglioside. There are 169 cases that could not be diagnosed in the study because of severe diseases failure to complete relevant examinations and physicians' poor recording in the cases. Medical staff in non-neurologic departments should strengthen the learning of common urgent and critical diseases related in clinics, while the neurologists provide more lectures to the junior physicians in the hospital.

\section{Limitations}

The analysis reflects retrospectively all of the urgent consultation cases within 4 years in our hospital except for the emergency department because of the special case recorded system. This analysis is limited by the discipline distribution of our hospital. The results may be not applicable to other hospitals. To know the current condition of urgent neurologic consultation, a large scale cases and multicenter study should be done in the future.

Acknowledgements: see in Title page

Conflict of interests

This study was approved by the First Affiliated Hospital Ethics Committee of XXX We have nothing to disclose financial or other conflict of interests.

References

1 Zhao C, Lee K, Do D. (2020) Neurology consults in emergency departments: Opportunities to streamline care.Neurol Clin Pract.10(2):149-15. doi:10.1212/CPJ.0000000000000712

2 Chirra M, Marsili L, Wattley L, et al.(2019) Telemedicine in Neurological Disorders: Opportunities and Challenges. Telemedicine and e-Health. 25(7):541-550. doi:10.1089/tmj.2018.0101.

3 Dall T, Storm M, Chakrabarti R et al.(2013) Supply and demand analysis of the current and future US neurology workforce. Neurology. 81(5):470-8. doi:10.1212/WNL.0b013e318294b1cf.

4 Zinchuk AV, Flanagan EP, Tubridy NJ et al.(2010)Attitudes of US medical trainees towards neurology education: "Neurophobia" - a global issue. BMC Med Educ. 10:49 doi:10.1186/1472-6920-10-49.

5 Bras PC, Barros A, Vaz S, Joao Sequeira, et al.(2018) Influence of weather on seizure frequency - clinical experience in the emergency room of a tertiary hospital.Epilepsy Behav. 86: 25-30. doi:10.1016/j.yebeh.2018.07.010.

6 Rodriguez-Antiguedad A., Matias-Guiu J., Hernandez-Perez M.A, et al.(2011)Neurophysiology training in the neurology specialist education program in Spain.Neurologia. 26(5):272-8. doi:10.1016/j.nrl.2010.11.003.

7 James G. Greene. (2018)Collaborative comanagement between neurohospitalists and internal medicine hospitalists decreases provider costs and enhances satisfaction with neurology care at an academic medical center. Neurohospitalist. 8(2):74-81. doi:10.1177/1941874417735173.

8 Wijdicks EFM, Hocker SE. (2018)A future for simulation in acute neurology. Semin Neurol. 38(4):465-470. doi:10.1055/s-0038-1666986. 
9 Sakashita A, Kizawa Y, Kato M, et al.(2018) Development of a standard for hospital-based palliative care consultation teams in Japan using a modified delphi method.J Pain Symptom Manage. 56(5):746-751. doi:10.1016/j.jpainsymman.2018.08.013.

10 Do DH, Siegler JE. (2018)Diagnoses and other predictors of patient absenteeism in an outpatient neurology clinic. Neurol Clin Pract. 8(4):318-326. doi:10.1212/CPJ.0000000000000488.

11 Ropper A. (2016)How to determine if you have succeeded at neurology residency. Ann Neurol. 79(3):33941. doi:10.1002/ana.24592.

12 Coban E, Mutluay B, Sen A, et al.(2016)Characteristics, diagnosis and outcome of patients referred to a specialized neurology emergency clinic: prospective observational study. Ann Saudi Med. 36(1):51-6. doi:10.5144/0256-4947.2016.51.

13 Roland B , Stephanie G, Audrey J, et al. (2018)Referral management: Which patients are deemed not appropriate for neurologic consultation, and what happens to them?[J].Clin Neurol Neurosurg. 173:15-19. doi:10.1016/j.clineuro.2018.05.025.

14 Morrish PK. (2015)Inadequate neurology services undermine patient care in the UK.BMJ. 350:h3284.doi:10.1136/bmj.h328

\section{Hosted file}

Table_1_Reasons_for_applying_for_urgent_consultation.doc available at https://authorea.com/users/ 316560/articles/446694-analysis-of-urgent-neurologic-in-hospital-consultations-in-a-largetertiary-hospital-center-in-china 